\title{
The Neuronal Activation of Deep Cerebellar Nuclei Is Essential for Environmental Enrichment-Induced Post- Stroke Motor Recovery
}

\author{
Qun Zhang1,\#, Jun-fa Wu ${ }^{1, \#, ~ Q i-l i ~ S h i ' ~}{ }^{2,3,4}$, Ming-yue Li $^{5}$, Chuan-jie Wang1, Xin Wang ${ }^{6}$, Wen- \\ yuan Wang ${ }^{4,1, *}$, Yi Wu, ${ }^{1, *}$
}

\begin{abstract}
${ }^{1}$ Department of Rehabilitation Medicine, Huashan Hospital, Fudan University, Shanghai, China. ${ }^{2}$ Stem Cell and Regenerative Medicine Laboratory, Ningbo Second Hospital, Zhejiang, China. ${ }^{3}$ University of Chinese Academy of Sciences, Beijing, China. ${ }^{4}$ Interdisciplinary Research Center on Biology and Chemistry, Shanghai Institute of Organic Chemistry, Chinese Academy of Sciences, Shanghai, China. ${ }^{5}$ Department of Rehabilitation Medicine, The Third Affiliated Hospital, Sun Yat-sen University, Guangzhou, China. ${ }^{6}$ Department of Rehabilitation, Clinical Medical College, Yangzhou University, Jiangsu, China
\end{abstract}

[Received September 22, 2018; Revised December 11, 2018; Accepted December 20, 2018]

\begin{abstract}
The level of cerebellar activity in stroke patients has been shown to correlate with the extent of functional recovery. We reasoned that the cerebellum may be an important player in post-stroke rehabilitation. Because the neurons in the deep cerebellar nuclei (DCN) represent virtually all of the output from the cerebellum, in this study, using environmental enrichment (EE) to promote rehabilitation, we investigated the influence of the optogenetic neuronal modulation of DCN on EE-induced rehabilitation. We found that neuronal inhibition of the DCN almost completely blocked motor recovery in EE treated mice, but the stroke mice with neuronal activation of the DCN achieved a similar recovery level as those in the EE treated group. No difference was observed in anxiety-like behavior. Moreover, $H$ tr2a in the DCN, the gene encoding 5-HT2A receptor, was shown to be a hub gene in the protein-protein interaction network identified using RNA-seq. This indicated that 5-HT2A receptor-mediated signaling may be responsible for $\mathrm{DCN}$-dependent functional improvement in $\mathrm{EE}$. We further verified this using the 5-HT2A receptor antagonist, MDL100907, to inhibit the function of 5-HT2A receptor in the DCN. This treatment resulted in impaired recovery in EE treated mice, who performed at a level as poor as the stroke-only group. Thus, this work contributes to an understanding of the importance of the DCN activation in EE-induced post-stroke rehabilitation. Attempts to clarify the mechanism of 5-HT2A receptor-mediated signaling in the DCN may also lead to the creation of a pharmacological mimetic of the benefits of EE-induced rehabilitation.
\end{abstract}

Key words: DCN, environmental enrichment, Htr2a, photothrombosis, optogenetics

Stroke is a leading cause of disability worldwide [1]. Numerous studies have attempted to enhance post-stroke functional recovery by modulating the activity of the perilesional and contra-lesional stroked areas. However, little clinical success has been made to-date [2]. Since poststroke deficiencies may occur in both adjacent and remotely connected brain areas [3], gaining control over other connected brain structures may represent new treatment possibilities.

The cerebellum is structurally connected to the sensorimotor cortex via the corticopontocerebellar tract (CPCT). Considerable evidence has implicated the

*Correspondence should be addressed to: Dr. Yi Wu, Huashan Hospital, Fudan University, Shanghai 200040, China. Email: wuyi@fudan.edu.cn; Dr. Wenyuan Wang, Shanghai Institute of Organic Chemistry, Chinese Academy of Sciences, Shanghai 201210, China. E-mail: wywang@ sioc.ac.cn. \#These_authors contributed equally to this study.

Copyright: () 2019 Zhang Q et al. This is an open-access article distributed under the terms of the Creative Commons Attribution License, which permits unrestricted use, distribution, and reproduction in any medium, provided the original author and source are credited. 
cerebellum as an important node in the brain networks that are altered following supratentorial stroke [4], which results in degeneration of the affected CPCT and increased robustness of the unaffected CPCT. Moreover, a positive correlation has been shown to exist between the activation of the affected cerebellum and the extent of functional recovery in stroke patients [5]. Chronic modulation of activity in the lateral cerebellar nucleus has been found to result in better post-stroke motor recovery in rodents [6]. Taken together, these observations indicate the cerebellum may be involved in the process of poststroke recovery, and neuromodulation of the deep cerebellar nuclei (DCN), which comprise the sole output pathway of the cerebellum, appears to be a promising approach for stroke rehabilitation. Despite this, the exact role of the DCN in rehabilitation has yet to be extensively described.

Environmental enrichment (EE) is a classical but effective therapeutic intervention for stroke rehabilitation in rodents. In comparison to a standard environment, EE provides comprehensive sensory, motor, cognitive and social stimulation [7]. In recent years, some clinical trials have also suggested that the use of EE in human stroke survivors, such as a mixed rehabilitation unit, may lead to health care cost savings due to its ability to improve better recovery and quality of life [8]. EE has been shown to facilitate post-stroke recovery through different molecular mechanisms in distinct brain areas, but the efficacy of EE as it relates to the cerebellum in stroke rehabilitation remains elusive. As previous studies have indicated that EE leads to an improvement in motor function in both cerebellar injury and Rett syndrome [9], the involvement of the cerebellum in EE-induced post-stroke recovery is deserving of further investigation.

In the present study, we aimed to examine the role of the DCN in EE-induced functional recovery following stroke. Specifically, we investigated whether optogenetic neuronal modulation of the DCN, the sole output nuclei of the cerebellum, influenced the efficacy of EE on poststroke recovery. We also sought to determine the molecular changes within the DCN after exposure to EE using RNA-seq. The candidate molecules revealed in this study will inform future attempts to create a pharmacological mimetic of EE, with the ultimate goal of improving post-stroke rehabilitation.

\section{MATERIALS AND METHODS}

\section{Animal preparation}

All experimental procedures were approved by the Animal Ethics Committee of the Fudan University (Shanghai, China). Male C57BL/6J mice (8-12 weeks of age, $22-25$ g body weight) were used in this study. All mice were housed in a 12:12 h light:dark cycle (with 08:00 to 20:00 as light-on hours), in a room with controlled temperature and humidity. All mice were anesthetized by intraperitoneal injection of $1 \%$ pentobarbital $(50 \mathrm{mg} / \mathrm{kg})$. During surgery, body temperature was maintained at $37^{\circ} \mathrm{C}$ with a heating pad.

\section{Photothrombotic stroke (PT)}

PT was induced unilaterally in the sensorimotor cortex. Briefly, anesthetized mice were placed in a stereotaxic device (RWD Life Sciences) with the skull exposed. The entire skull was then covered by an opaque template which exposed a circular region of the left sensorimotor cortex (coordinates: rostral to caudal: 2.5 to $1.5 \mathrm{~mm}$, medial to lateral: 0 to $4 \mathrm{~mm}$, relative to bregma). Immediately after intravenous injection of $0.1 \%$ Rose Bengal $(0.01 \mathrm{~mL} / \mathrm{g}$ body weight, dissolved in $0.9 \% \mathrm{NaCl}$, Sigma), the sensorimotor cortex was illuminated with a $532 \mathrm{~nm}$ green laser beam $(50 \mathrm{mw})$ for $10 \mathrm{~min}$ at maximum output.

\section{Stereotaxic Surgery}

\section{Virus injection and Optic Fiber Implantation}

Anesthetized mice were fixed into a stereotaxic device using ear bars. To prevent any corneal damage, ophthalmic ointment was used. A burr hole was drilled into the right side of the cerebellum, and a glass electrode filled with virus was aimed at the DCN. The intended mouse brain DCN stereotactic coordinates, as previously described [10], were as follows: $\mathrm{AP}=-6.0 \mathrm{~mm}$ from bregma, $\mathrm{ML}=1.5 \mathrm{~mm}$ from the midline, $\mathrm{DV}=-3.5 \mathrm{~mm}$ from the skull surface. $200 \mathrm{~nL}$ of rAAV-hsyn-eNPHR3.0mcherry-WPRE-PA (NPHR gene expression group) or rAAV-hsyn-hChR2-mcherry-WPRE-PA (ChR2 gene expression group) were randomly assigned to be injected into the DCN at a rate of $20 \mathrm{~nL} / \mathrm{min}$. After injection, the electrode filled with the virus was left in place for at least 10 min before it was withdrawn, and an optic fiber cannula $(200 \mu \mathrm{m})$ was then slowly implanted into the injection site. The optical fiber cannula was fixed to the skull with dental cement. The mice were returned to the animal facility to recover for 2 weeks before PT surgery.

\section{Stimulation Paradigm}

Mice received photostimulation beginning on the 7th day post-PT surgery. Stroked mice were placed in an empty cage to allow for free movement with the laser cable connected to the fiber cannula. One session of stimulation consisted of three 1 min stimulations separated by $3 \mathrm{~min}$ rest intervals. All of the mice, including the sham- 
stimulated stroked mice, received daily (7 days/week) stimulations for 2 weeks post-stroke. In the ChR2 gene expression group, a $473 \mathrm{~nm}$ blue laser was controlled by a driver (Thorlabs, DC2200), and mice were stimulated with a laser set to $10 \mathrm{~Hz}$, with $20 \mathrm{msec}$ light pulses. In the NPHR gene expression group, a $594 \mathrm{~nm}$ yellow laser was controlled by the same driver as above, whereas mice were stimulated with direct current. The laser power operated with a range of $1.2-1.4 \mathrm{~mW}$, which was measured by a power meter before implantation. Stimulations were performed in the morning between 12:00 and 13:00. Sham stimulation (SS) was conducted without turning on the laser.

The day after the final behavioral test, mice were anesthetized and then transcardially perfused with $4 \%$ paraformaldehyde. The regions of virus injection were confirmed under a fluorescence microscope. Data from mice with an absence of ChR2 or NPHR gene expression were excluded from the group.

\section{Cannula Implantation}

A burr hole was drilled on the right side of the cerebellum. Another two holes were drilled near the implantation point for screw insertion. The cannula was then inserted into the DCN and fixed with dental cement. For subsequent pump installation, a $2 \mathrm{~cm}$ long PE50 catheter, filled with sterilized artificial cerebrospinal fluid (aCSF), was inserted into the angle arm of the cannula, and the other end of this catheter was sealed for later pump connection. The sealed end of the catheter was put into the pocket made between the shoulder blades of the mice. Mice were allowed to recover for 14 days before they received PT surgery.

\section{Osmotic Pump Installation}

Mice received osmotic pump installation on the $7^{\text {th }}$ day post-PT surgery. Osmotic pumps were filled with MDL100907 (2.5 mM, $180 \mu \mathrm{L}$, Tocris) and kept in sterile distilled water at $37^{\circ} \mathrm{C}$ overnight before installation [11]. In stroked mice, an incision between the shoulder blades was made and an osmotic pump was connected to the free end of the PE50 catheter, which was left in the pocket in advance. The skin was then tightly stitched with a 5-0 suture thread.

\section{Housing conditions}

After PT surgery, mice were randomly assigned to one of the following groups: sham group, PT group and PT with $\mathrm{EE}$ group $(\mathrm{PT}+\mathrm{EE})$. Mice in the sham and $\mathrm{PT}$ groups were housed in standard cages $(294 \times 190 \times 130 \mathrm{~mm})$ for $7-21$ days after stroke, with five mice per cage. Mice in the
$\mathrm{PT}+\mathrm{EE}$ group were housed in larger cages $(545 \times 395 \times$ $200 \mathrm{~mm}$ ), containing various different objects (e.g., chains, runners, ladders, pipelines, boxes, etc.) which were changed every day.

\section{Behavioral tests}

All of the behavioral tests took place between 09:00 and 11:00 and were conducted under the illumination of a 60 $\mathrm{W}$ light bulb affixed $2.4 \mathrm{~m}$ above each apparatus. Mice were brought to the testing room for acclimation $1 \mathrm{~h}$ prior to each test. After completion of the behavioral tests, mice were returned to their respective environments for $60 \mathrm{~min}$, after which they were sacrificed for brain tissue. Each apparatus was cleaned with $75 \%$ ethanol after removal of each animal.

\section{Accelerating rotarod}

The rotarod apparatus (gradual acceleration of 5-40 revolutions per minute [RPM] within $90 \mathrm{~s}$ ) was used to assess motor performance. The pre-training test (in order to establish a performance baseline), was conducted at a linear accelerating speed of 5-40 RPM for three trials per day for 3 consecutive days. The rotarod test was conducted on the 7th, 14th, and 21st day post-PT surgery, or the 14th day after fiber or cannula implantation (Fig. $1 \mathrm{~A}, 3 \mathrm{~A}, 6 \mathrm{~B})$. Each trial lasted a maximum of $5 \mathrm{~min}$, and a 3 min rest interval between trials was allowed to avoid fatigue. The latencies to falling off the rod for each mouse in each trial were recorded, and the average latencies were calculated as the final result. The trial was terminated when mice spun around the rod through three complete revolutions.

\section{Cylinder test}

Mice were placed in a transparent glass cylinder (diameter $9.5 \mathrm{~cm}$, height $16 \mathrm{~cm}$ ), with two mirrors angled $45^{\circ}$ positioned behind it. The spontaneous rearing and forepaw contacts on the cylinder of each mouse were recorded for 5 min, including contacts with the left paw only, right paw only and both paws simultaneously. The cylinder test was conducted on the 7th, 14th, and 21st day post-PT surgery, or the 14th day after fiber or cannula implantation (Fig. 1A, 3A, 6B). Data were analyzed using asymmetry, which was calculated as follows: (left + $0.5 \times$ both $) /($ right + left + both $) \times 100 \%$.

\section{Rung walking test}

The ladder rung apparatus was composed of two plexiglass walls $(69.5 \mathrm{~cm} \times 15 \mathrm{~cm})$, which were spaced 5 $\mathrm{cm}$ apart to allow for the passage of a mouse but prevented 
its turning around. A total of 31 plastic bars with a diameter of $0.1 \mathrm{~cm}$ were arranged at a distance of $1 \mathrm{~cm}$ apart and inserted into the holes on the walls. The entire apparatus was placed on two holders, $17 \mathrm{~cm}$ above the ground. Before the PT surgery, mice were trained to cross the ladder for three consecutive trials. The test was conducted on the 7th, 14th, and 21st day post-PT surgery, or the 14th day after fiber or cannula implantation (Fig. $1 \mathrm{~A}, 3 \mathrm{~A}, 6 \mathrm{~B})$. With a camera positioned at a slight ventral angle to the apparatus, we were able to record the four limbs of the walking mouse at the same time. The total number of steps and the number of errors made by the right forelimb were then recorded.

\section{Elevated plus maze test (EPM)}

Anxiety-like behaviors were recorded for $5 \mathrm{~min}$ in the EPM. Mice facing one of the closed arms were placed in the central neutral zone at the beginning of the test. The cumulative percentage of time spent in the open and closed arms were subsequently recorded. The test was only conducted on the 21 st day post-PT surgery.

\section{Data analysis}

Data from motor behavioral tests were analyzed using a two-way ANOVA with a Tukey's multiple comparisons post-test. EPM data were analyzed using a one-way ANOVA with a Tukey's multiple comparisons post-test. An unpaired two-tailed $t$-test was used to analyze qPCR data. All the analysis and statistics were performed using
GraphPad Prism Version 6.0. In all cases, a $p$ value less than or equal to 0.05 was considered significant. All data are shown as the mean values \pm standard error of the mean (SEM).

\section{RNA-seq library generation and sequencing}

Total RNA was isolated from the right DCN using TRizol (Invitrogen, USA) in the PT and PT+EE groups. To generate RNA sequencing libraries, a TruSeq RNA Library Preparation Kit v2 (Illumina) was used, following the manufacturer's instructions. Pair-end 150 bp sequencing was then performed on a HiSeq x102500 Illumina at the WuXi NextCODE Corporation.

\section{Quantitative real-time PCR}

Total RNA (1 ug) from samples in the PT and PT+EE groups were extracted and converted into cDNA with the Hifair $^{\mathrm{TM}}$ III 1st Strand cDNA Synthesis SuperMix Kit (YEASEN) in a total volume of $20 \mu \mathrm{L}$. Quantitative PCR was performed on the Quantstudio ${ }^{\mathrm{TM}}$ Real-Time PCR System (Thermo Fisher) in a final reaction volume of 10 $\mu \mathrm{L}$, using $50 \mathrm{ng}$ of the cDNA template from each sample. GAPDH served as the housekeeping control. Reactions were performed in triplicate with a minimum of three independent runs. Data were analyzed using the $\Delta \Delta \mathrm{Ct}$ method on Quantstudio ${ }^{\mathrm{TM}}$ Real-Time (RT) PCR software (Thermo Fisher). Primer sequences are displayed in Table1.

Table 1. Primer sequences used for the validation of cerebellum-associated gene expression amongst upregulated genes.

\begin{tabular}{lll}
\hline Gene & Forward primer & Reverse primer \\
\hline Ngb & CTGCTGCCTCTCTTCCAGTA & AGCTGGTCAGGTACTCCTCC \\
Gabrq & AAATGTGCAGGATGGCCTGA & TGGCCAATTGAGTCTGGCTT \\
Htr1a & CAGGGCAACAACACCACAAC & CGTTGGAGAGGCCAGTATCG \\
Htr2a & GCTCTGTGCCGTCTGGATT & CGGCTATGGTGAATGGGGTT \\
Chrm1 & TGCTGGTGCTCATCTCCTTC & CCCATGAGCAGGTATGTGGT \\
\hline
\end{tabular}

\section{RNA-seq data processing}

The sequencing reads were aligned to the mouse reference genome GRCm38 using TopHat and Bowtie2. Cufflinks were then used to assemble the aligned sequences into transcript isoforms and identify the transcription start sites, using the mouse transcript annotations from Ensembl 81 as a guide. Cuffdiff was used to calculate the FPKM values and test for differential expression. CummeRbund was employed for post-processing of the results in $\mathrm{R}$. The differentially expressed genes were expressed as fold-change with Benjamini-Hochberg FDR corrected q-values $<0.05$. Hierarchical clustering and heat maps were generated in $\mathrm{R}$.

\section{RESULTS}

\section{Environmental enrichment promotes motor recovery following photothrombotic stroke}

A rotarod test was used to assess motor coordination ability (Fig. 1B). Significant effects of time $(F[2$, $243]=52.90, \quad p<0.0001), \quad$ treatment $\quad(F[5,243]=39.80$, $p<0.0001)$ and time $\times$ treatment interaction $(F[10$, $243]=8.84, p<0.0001)$ were observed using a two-way 
ANOVA test with repeated measures. Upon further analysis with a Tukey's multiple comparisons test, we found no obvious distinction between groups in the pretrial training $(p=0.78)$. However, compared to the sham group, serious motor deficiencies were observed in the PT group on the 7 th day post-PT, with an approximately $60 \%$ reduction in the rotarod travel time. As EE started from the 7th day post-stroke onwards, better performance was observed in the PT+EE group compared to the PT group on both the 14th $(p=0.004)$ and 21 st $(p<0.0001)$ day postPT $\left(\mathrm{n}_{\text {sham }}=9, \mathrm{n}_{\mathrm{PT}}=17, \mathrm{n}_{\mathrm{PT}+\mathrm{EE}}=17\right)$.
A

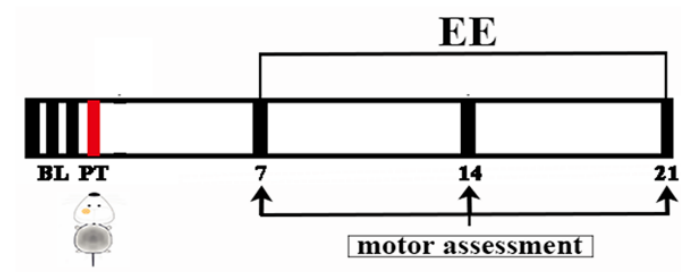

B

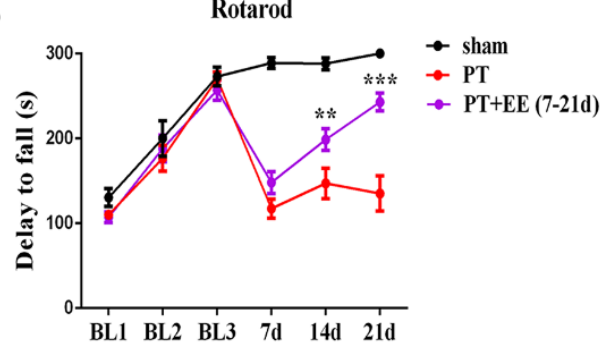

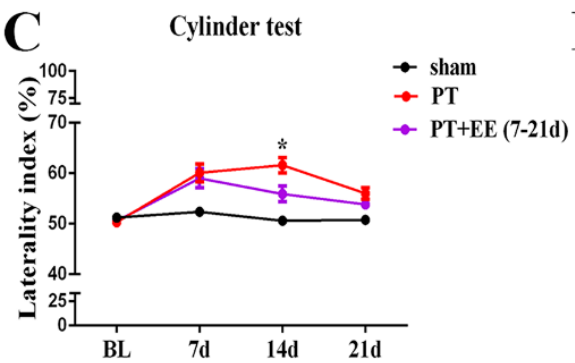
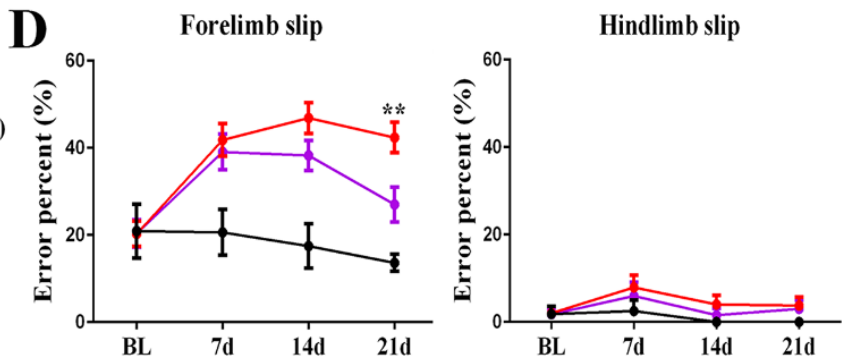

Figure 1. Effects of EE on motor recovery after PT. (A) Time course of behavioral tests. Motor assessments included the rotarod, cylinder and rung walking tests. BL: baseline data for rotarod. (B) Time course of the latency to fall off the rotarod. (C) The percentage of laterality in the cylinder test. (D-E) The percentage of limb slips in the rung walking test, for both forelimb and hindlimb. Data are expressed as the mean \pm SEM. ${ }^{*}$ PT vs. PT+EE groups. ${ }^{*} p \leq 0.05 ;{ }^{* *} p \leq 0.01 ;{ }^{* * *} p \leq 0.001$.

Additionally, the cylinder test (Fig. 1C) and rung walking test (Fig. 1D-E) were conducted, which more sensitively detect impairments in fine motor skills. In the cylinder test, a two-way ANOVA with repeated measures indicated significant effects of time $(F[3,124]=15.00$, $p<0.0001)$, treatment $(F[2,124]=18.60, p<0.0001)$ and time $\times$ treatment interaction $(F[6,124]=3.98, p=0.001)$ among the three groups. On the 7th day post-PT, a marked deficit in the use of the right forelimb and a higher reliance on the contralateral left forelimb was observed in stroked mice of both PT and EE groups. However, on the 14th day post-PT the EE treated group tended to touch the cylinder wall more often with both paws ( $p=0.028)$, and less often with their left forelimb only $(p=0.002)$, in comparison to the PT group. Both PT and PT+EE groups recovered gradually, and by the 21 st day post-stroke the two groups displayed equal paw placement times $(p=0.16$; $\mathrm{n}_{\text {sham }}=8, \mathrm{n}_{\mathrm{PT}}=13, \mathrm{n}_{\mathrm{PT}+\mathrm{EE}}=13$ ).

Similarly, a two-way ANOVA with repeated measures indicated significant effects of time $(F[3$, $99]=4.94, p=0.003)$, treatment $(F[2,99]=14.70, p<0.0001)$ and time $\times$ treatment interaction $(F[6,99]=2.28, p=0.04)$ on forelimb placement among the three groups (Fig. 1D). No significant difference was found in hindlimb slips between the PT and PT+EE groups (Fig. 1E). However, performance in the rung walking test was better in the $\mathrm{PT}+\mathrm{EE}$ group compared to the PT group. An obvious increase in forelimb slips was observed on the 7th day post-PT. Although no statistically significant difference was found between the PT and PT+EE groups on the 14th day post-PT, a faster and more robust recovery was observed in the EE treated group on the 21 st day poststroke, as assessed by a Tukey's multiple comparisons test $\left(p=0.01 ; \mathrm{n}_{\text {sham }}=5, \mathrm{n}_{\mathrm{PT}}=10, \mathrm{n}_{\mathrm{PT}+\mathrm{EE}}=10\right)$.

Despite the fact that the above data collectively suggested a facilitation of motor recovery with EE, the level of improvement in these three behavioral tests appeared to be different; compared to the cylinder and rung walking tests, EE resulted in more robust and faster improvement in the rotarod test. 
A

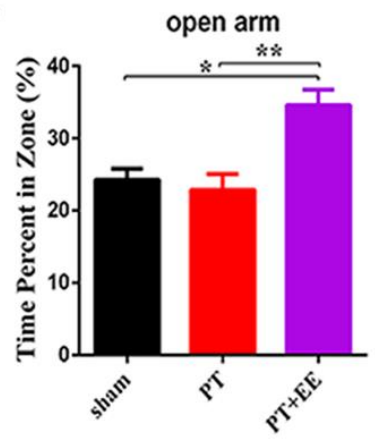

B

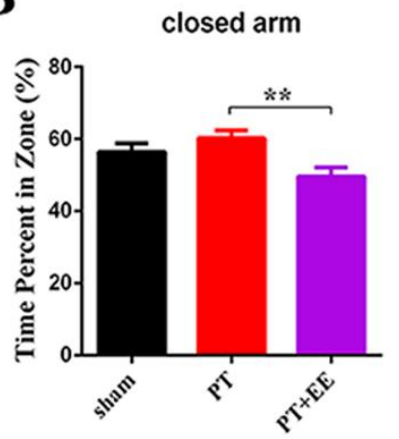

C

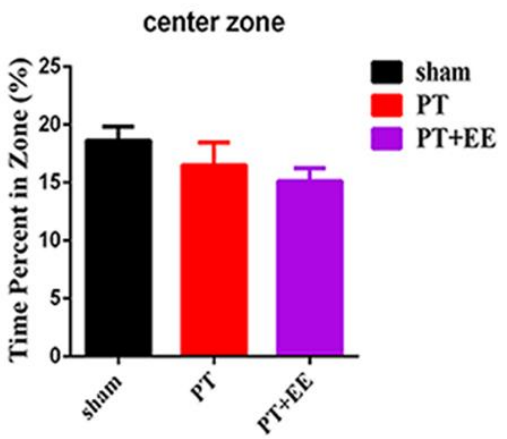

Figure 2. Effects of EE on anxiety-like behavior after PT. (A-C) The percentage of time mice spent in the open arm (A), closed arm (B) and center zone $(\mathbf{C})$ of the elevated plus maze in each group. Data are expressed as the mean $\pm \mathrm{SEM} ;{ }^{*} p \leq 0.05 ;{ }^{* *} p \leq 0.01$.

\section{Environmental enrichment attenuates anxiety-like behavior}

In order to evaluate the exploration ability or anxiety level of mice, we analyzed EPM data by comparing the time spent in each maze zone (Fig. 2). Compared to the PT group, the PT+EE group spent more time in the open arm (Fig. 2A), and less in the closed arm, as assessed by a one-way ANOVA (Fig. 2B; open arm: $F[2,30]=9.78$, $p=0.001$; closed arm: $F[2,30]=5.84, p=0.01)$. This indicated an attenuating role of $\mathrm{EE}$ on anxiety-like behavior. However, as no remarkable differences were observed between PT and sham groups, the PT stroke model appeared to have no effect on anxiety-like behavior. Additionally, the time spent in the center zones (Fig. 2C) was virtually identical among the three groups $\left(F[2,30]=0.96, \quad p=0.39 ; \quad \mathrm{n}_{\text {sham }}=7, \quad \mathrm{n}_{\mathrm{PT}}=13\right.$, $\left.\mathrm{n}_{\mathrm{PT}+\mathrm{EE}}=13\right)$.

\section{Repeated optogenetic DCN stimulation modulates environmental enrichment-induced motor recovery}

To further investigate the role of the DCN in EE-induced recovery after stroke, we used optogenetics to modulate DCN activity, allowing us to simultaneously observe the resulting behavioral changes (Fig. 3B-C).

In the rotarod test (Fig. 3 D-E), significant effects of time $(F[6,293]=68.60, p<0.0001)$, treatment $(F[5$, 293] $=11.30, p<0.0001)$ and time $\times$ treatment interaction $(F[30,293]=3.52, p<0.0001)$ were found, as assessed using a two-way ANOVA with repeated measures. Upon further analysis with a Tukey's multiple comparisons test, we found that, compared to the PT+SS group, repeated DCN activation resulted in an increased latency to fall in the $\mathrm{PT}+\mathrm{ChR} 2$ group $(p=0.01)$ on the 21 st day post-PT, which was as long as that of the PT+EE+SS group $(p=0.56)$. However, compared to the performance of the PT+EE+SS group (14th day post-PT, $p=0.001 ; 21$ st day post-PT, $p<0.0001$ ), when the PT+EE+NPHR group was given DCN inhibition, mice exhibited significantly slower and poorer recovery rates, at the same level as those observed in the PT+SS group (14th day post-PT, $p=0.84 ; 21$ st day post-PT, $p=0.74)$. No significant difference was found between the PT+SS and PT+NPHR groups (14th day post-PT, $p=0.32 ; 21$ st day post-PT, $p=0.90 ; \mathrm{n}_{\mathrm{PT}+\mathrm{SS}}=9$, $\mathrm{n}_{\mathrm{PT}+\mathrm{EE}+\mathrm{SS}}=10, \mathrm{n}_{\mathrm{PT}+\mathrm{NPHR}}=9, \mathrm{n}_{\mathrm{PT}+\mathrm{ChR} 2}=7, \mathrm{n}_{\mathrm{PT}+\mathrm{EE}+\mathrm{NPHR}}=8$,

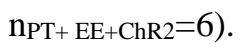

In the cylinder test (Fig. 3F-G), the effects of time $(F[4,241]=29.30, p<0.0001)$, treatment $(F[5,241]=2.74$, $p=0.02)$ and time $\times$ treatment interaction $(F[20,241]=1.40$, $p=0.12$ ) were also analyzed using a two-way ANOVA with repeated measures. Upon further analysis with a Tukey's multiple comparisons test, the $\mathrm{PT}+\mathrm{ChR} 2$ group was found to have a better rate of recovery than the PT+SS group on the 14th day post-PT ( $p=0.001)$, although not better than that of the PT+EE+SS group $(p=0.45)$. However, by the 21 st day post-PT, a similar performance was observed in the $\mathrm{PT}+\mathrm{ChR} 2$ and $\mathrm{PT}+\mathrm{SS}$ groups $(p=0.94)$. Like the observed rotarod behavior, repeated DCN activation in the $\mathrm{PT}+\mathrm{EE}+\mathrm{ChR} 2$ group did not facilitate significant changes in forelimb asymmetry when compared with results from the PT+EE+SS group (14th day post-PT, $p=0.99 ; 21$ st day post-PT, $p=0.99)$. Nevertheless, when the PT+EE+NPHR group was treated with DCN inhibition, mice exhibited a robust deterioration in the use of the paralyzed forelimb compared to that of the PT+EE+SS group by the 14th day post-PT $(p=0.01)$, which was as poor as that observed in the PT+SS group (14th day post-PT, $p=0.88 ; 21$ st day post-PT, $p=0.35$ ). Likewise, no significant difference was found between the PT+SS and PT +NPHR groups $\left(14^{\text {th }}\right.$ day post-PT, $p>0.99 ; 21 \mathrm{st}$ day post-PT, $p>0.99$; $\mathrm{n}_{\mathrm{PT}+\mathrm{SS}}=13, \quad \mathrm{n}_{\mathrm{PT}+\mathrm{EE}+\mathrm{SS}}=13, \quad \mathrm{n}_{\mathrm{PT}+\mathrm{NPHR}}=13, \quad \mathrm{n}_{\mathrm{PT}+\mathrm{ChR} 2}=7$, $\mathrm{n}_{\mathrm{PT}+\mathrm{EE}+\mathrm{NPHR}}=7, \mathrm{n}_{\mathrm{PT}+\mathrm{EE}+\mathrm{ChR} 2}=7$ ). 
A

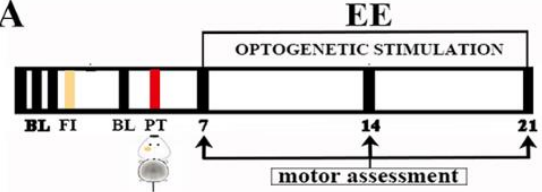

D

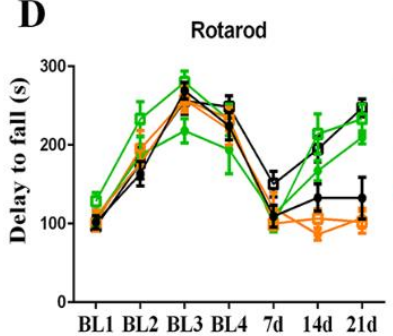

F Cylinder test

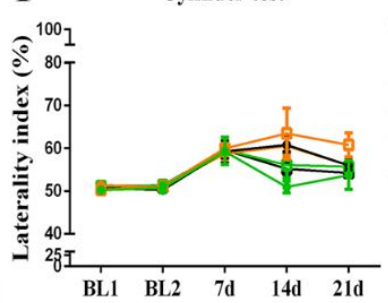

H
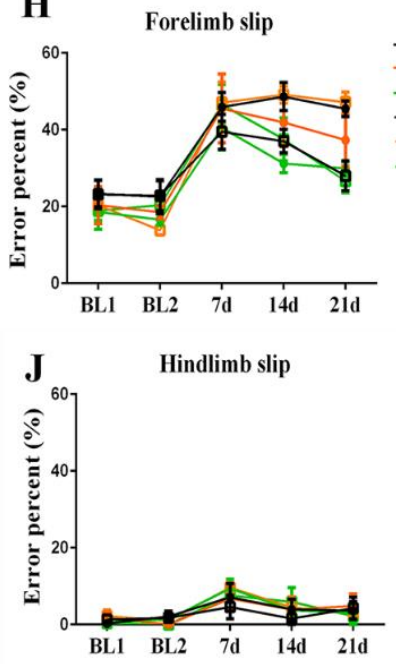

B

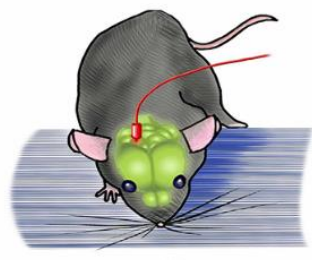

E

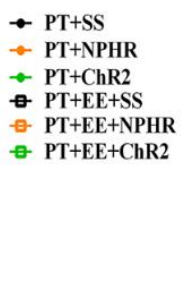

$\rightarrow \mathrm{PT}+\mathrm{SS}$

$\rightarrow$ PT + NPHR

$\rightarrow \mathrm{PT}+\mathrm{ChR} 2$

* PT $+\mathrm{EE}+\mathrm{SS}$

-7. PT + EE + NPHR

- $\mathrm{PT}+\mathrm{EE}+\mathrm{ChR2}$

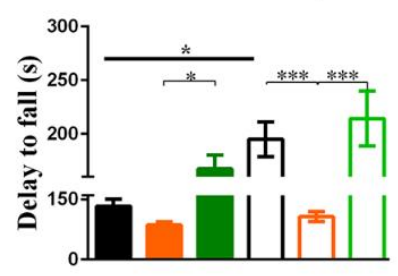

G
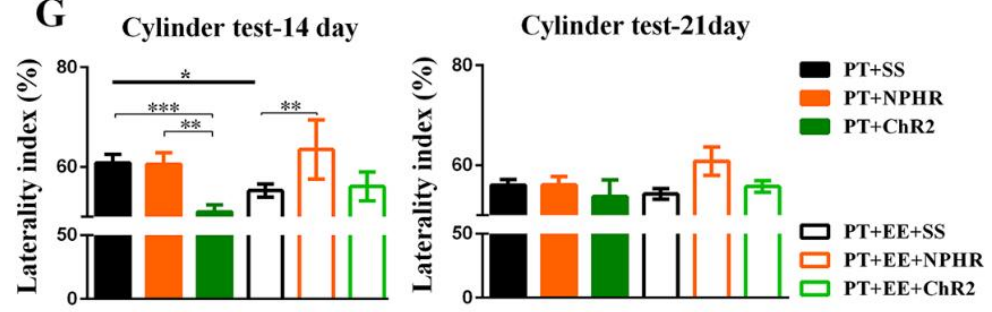

Rotarod-21 day

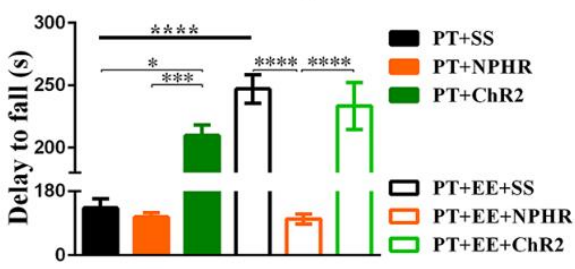

C

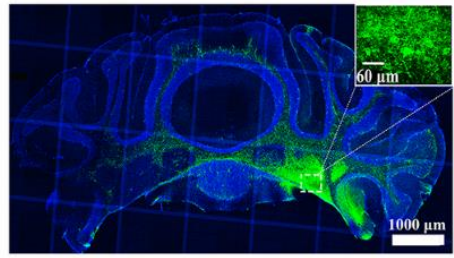

I
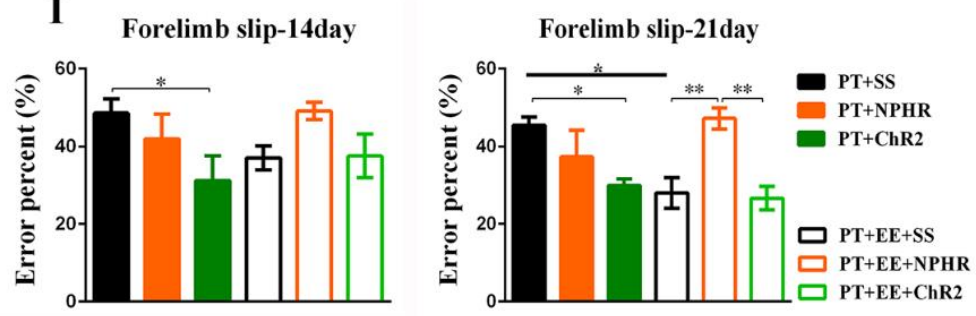

K Hindlimb slip-14day

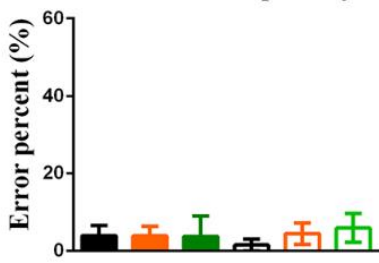

Hindlimb slip-21day

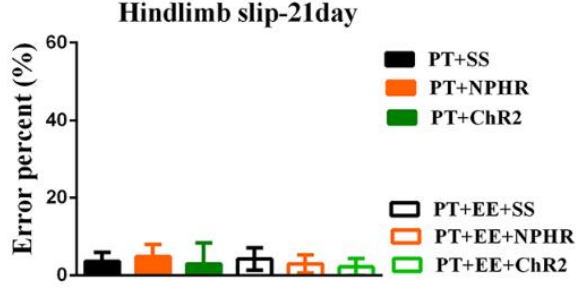

Figure 3. Effects of optogenetic DCN stimulation on motor recovery. (A) Time course of behavioral tests after optogenetic stimulation of the DCN. Motor assessments included the rotarod, cylinder and rung walking tests. BL: baseline data for rotarod. FI: fiber implantation. (B) Schematic depiction of the optogenetic DCN stimulation used to modulate motor function. (C) Representative image of viral injection location targeted to the DCN. Positive expression of ChR2-GFP was observed in the DCN (100X magnification, scale bar $=1 \mathrm{~mm}$ ). The morphology of the neurons (green) was shown in the inserted image (400X magnification, scale bar $=60 \mu \mathrm{m}$ ). (D) Time course of the latency to fall off the rotarod in each group. (E) Histogram of the falling off latency in each group on the 14th and 21th day after stroke. (F) The percentage of laterality in the cylinder test in each group. (G) Histogram of the laterality percentage in each group on the 14th and 21th day after stroke. (H and J) The percent of forelimb slips (H) and hindlimb slips (J) in the rung walking test in each group. (I, K) Histogram of the limb slips percentage in each group on the 14 th and 21 th day after stroke. Data are expressed as the mean \pm SEM. ${ }^{*} p \leq 0.05 ;{ }^{* *} p \leq 0.01 ;{ }^{* * *} p \leq 0.001 ;{ }^{* * * *} p \leq 0.0001$. 

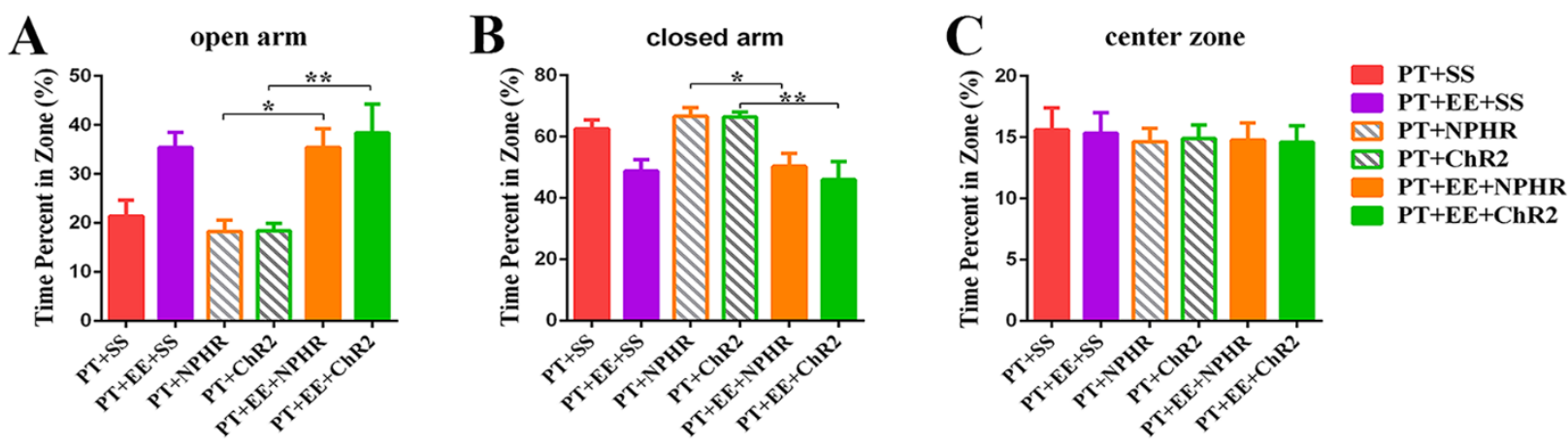

Figure 4. Effects of optogenetic DCN stimulation on anxiety-like behavior after PT. The percentage of time spent in the open $\operatorname{arm}(\mathbf{A})$, closed arm (B) and center zone (C) of the elevated plus maze. Data are expressed as the mean \pm SEM; ${ }^{*} p \leq 0.05 ;{ }^{* * *} p \leq 0.01$.

In the rung walking test (Fig. $3 \mathrm{H}-\mathrm{I})$, the effects of time $(F[4,190]=46.30, p<0.0001)$, treatment $(F[5,190]=3.97$, $p=0.002)$ and time $\times$ treatment interaction $(F[20,190]=1.34$, $p=0.14$ ) were again analyzed using a two-way ANOVA with repeated measures, with further analysis by a Tukey's multiple comparisons test. Repeated DCN activation resulted in more improvement in the $\mathrm{PT}+\mathrm{ChR} 2$ group than in the PT+SS group on the 21st day post-PT $(p=0.02)$, which was equal to that of the PT+EE+SS group $(p=0.89)$. Moreover, repeated DCN activation in the $\mathrm{PT}+\mathrm{EE}+\mathrm{ChR} 2$ group did not facilitate improved recovery relative to the PT+EE+SS group (14th day post-PT, $p>0.99 ; 21$ st day post-PT, $p>0.99)$. However, DCN inhibition in the PT+EE+NPHR group resulted in a slower and poorer recovery compared to the $\mathrm{PT}+\mathrm{EE}+\mathrm{SS}$ group on the 21 st day post-PT ( $p=0.01$ ), which was identical to that observed in the PT+SS group ( $p=0.99)$. No significant differences were found in the number of hindlimb slips

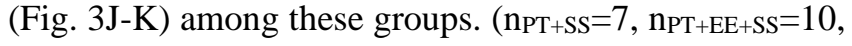
$\left.\mathrm{n}_{\mathrm{PT}+\mathrm{NPHR}}=7, \mathrm{n}_{\mathrm{PT}+\mathrm{ChR} 2}=7, \mathrm{n}_{\mathrm{PT}+\mathrm{EE}+\mathrm{NPHR}}=7, \mathrm{n}_{\mathrm{PT}+\mathrm{EE}+\mathrm{ChR} 2}=7\right)$.

\section{Repeated optogenetic DCN stimulation has no effect on the environmental enrichment-induced attenuation of anxiety-like behavior}

To identify the potential involvement of the DCN in the EE-induced attenuation of anxiety-like behavior, we again used optogenetics to modulate DCN activity and recorded the time spent in the EPM test (Fig. 4A-C). In line with previous results, we did not observe a significant effect of DCN stimulation, as assessed using a one-way ANOVA, on the amount of time spent in each maze area. The PT+EE groups receiving repeated optogenetic DCN stimulation, either activation or inhibition, all spent significantly more time in the open arm of the maze (Fig. 4A), and decreased time in the closed arm of the maze (Fig. 4B), compared to the PT group (open arm: $F[5,37]=6.99, p=0.0001$; closed arm:
$F[5,37]=6.38, p=0.0002)$. There were no significant differences found among all groups in the amount of time spent in the center zone of the maze (Fig. 4C; $F[5$, 37 $]=0.084, p=0.99 ; n_{P T+S S}=8, n_{P T+E E}+S S=7, n_{P T+N P H R}=7$, $\mathrm{n}_{\mathrm{PT}+\mathrm{ChR} 2}=7, \mathrm{n}_{\mathrm{PT}+\mathrm{EE}+\mathrm{NPHR}}=7, \mathrm{n}_{\mathrm{PT}+\mathrm{EE}+\mathrm{ChR} 2}=7$ ).

\section{Htr2a is a hub gene that is expressed differentially in mice exposed to environmental enrichment}

To investigate whether the molecular pathways associated with the DCN were altered in mice subjected to EE, we performed RNA-seq on a total of six DCN samples comprising three independent biological replicates from the PT and PT+EE groups. Hierarchical clustering (Fig. 5 A-B) revealed that samples clustered in agreement with the group assignment, with a total of 1,384 differentially expressed genes found between the two groups. The list of genes comprised both annotated mRNAs and unknown transcripts. The top 30 upregulated and downregulated mRNAs in each group are displayed in Figure 5C. Compared to the downregulated genes in the PT+EE group relative to the PT group $(\mathrm{n}=557)$, which ranged from 1.23 to 40.90 -fold, more robust changes were observed amongst the upregulated genes $(n=809)$, which ranged from 1.23 to 120.26 -fold over the PT group. Therefore, we used the top 200 genes from the upregulated list for further protein-protein interaction (PPI) network construction. Strikingly, we found that $41.5 \%(17 / 41)$ of genes in the most connected module were related to normal functioning of the cerebellum, which are denoted as red dots in Figure 5D. Notably, $H t r 2 a$, which encodes 5-HT $T_{2 A}$ receptor, a sub-type of serotonin receptors responsible for motor coordination in the cerebellum, tended to be a hub gene in the PPI network. To validate the RNA-seq data, we further tested the selected mRNAs from the list of upregulated genes against the same RNA as that from RNA-seq experiments, which showed consistent changes with the RNA-seq data 
(Fig. 5E). Together, this experiment identified molecular changes related to DCN function in EE treated samples, allowing us to select Htr2a for further experimental examination.

Next, we examined the expression levels of Htr2a in new DCN samples from sham, PT and PT+EE groups (five samples with three replicates in each group) via RT-
PCR. Using a two-tailed $t$-test, a significantly $(\sim 50 \%)$ higher Htr $2 a$ expression was observed in the PT+EE group compared to the PT group (Fig. $6 \mathrm{~A} ; t=2.22, d f=26$, $p=0.04$ ), whereas no difference was observed between the sham and PT+EE groups $(t=0.57, d f=23, p=0.57)$.

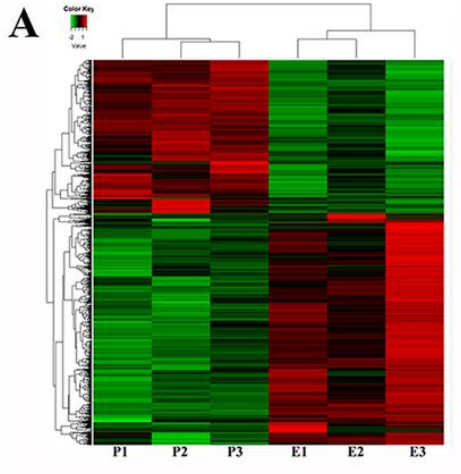

C

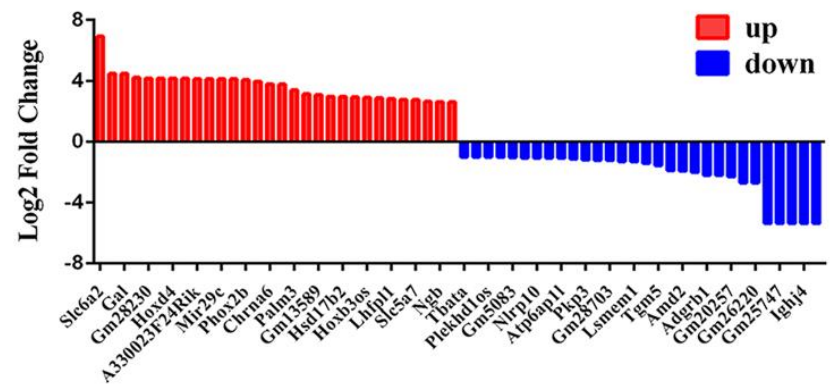

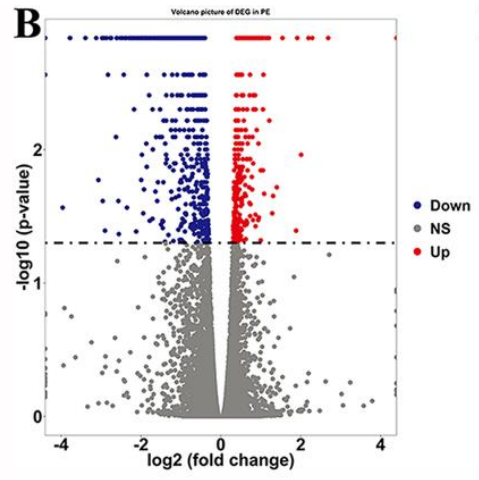

D

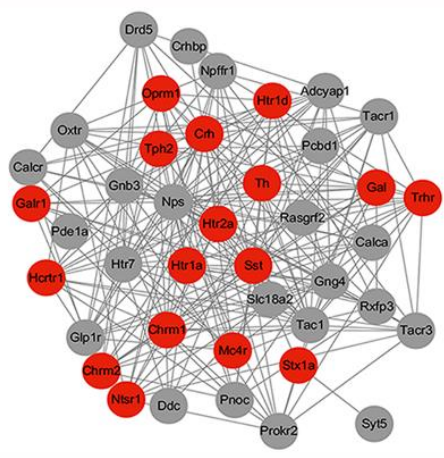

E

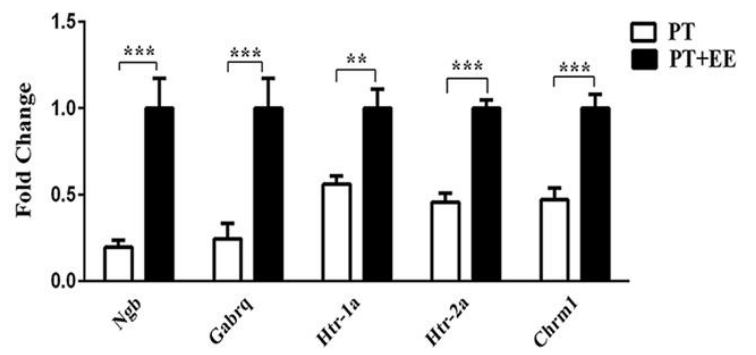

Figure 5. Hierarchical clustering of transcripts in the DCN of PT and PT+EE groups. (A) Heat map of differentially expressed genes in the PT vs. the PT+EE group. Each group included three independent biological replicates. Red: high, Green: low, log scale from -2 to +1 . (B) Volcano plots of significant differential expression of mRNAs in the PT versus the PT+EE group. The X-axis represents $\log _{2}$-fold change, and the y-axis represents $-\log 10$ ( $p$-value). Blue dots: genes that were lower in the PT+EE group compared to the PT group. Red dots: genes that were higher in the PT+EE group compared to the PT group. Grey dots: genes that were unchanged in the PT+EE and PT groups. (C) Representative distribution of genes in the high expression subset and low expression subset in the PT+EE vs. PT group. Blue column: genes that were lower in the PT+EE group compared to the PT group. Red column: genes that were higher in the PT+EE group compared to the PT group. (D) PPI (protein-protein interaction) network of the upregulated genes. The red nodes are known cerebellar target genes. (E) RT-PCR validation of the expression of cerebellarassociated genes, from the list of upregulated genes. Data are expressed as the mean fold-change \pm SEM from three separate experiments, conducted in triplicate. ${ }^{* *} p \leq 0.01 ;{ }^{* * *} p \leq 0.001$.

\section{The 5-HT $T_{2 A}$ receptor antagonist MDL100907 inhibits environmental enrichment-induced motor recovery following stroke}

We next made use of $M D L 100907$, a selective 5-HT $2 A$ receptor antagonist, in order to inhibit the receptor's activity in the affected DCN, and further examine the role of the DCN 5-HT $T_{2 A}$ receptor in EE-induced recovery after stroke (Fig. 6C).
In the rotarod test (Fig. 6D-E), significant effects of time $(F[6,133]=77.70, \quad p<0.0001)$, treatment $(F[3$, $\left.\left.{ }_{133}\right]=10.80, p<0.0001\right)$ and time $\times$ treatment interaction $(F[18,133]=3.04, p=0.0001)$ were observed using a twoway ANOVA with repeated measures. Further analysis with a Tukey's multiple comparisons test indicated that, compared to the PT+EE+PBS group, the latencies to fall were considerably decreased in the PT+PBS, $\mathrm{PT}+M D L 100907$ and $\mathrm{PT}+\mathrm{EE}+M D L 100907$ groups. Even given the presence of EE treatment, no improvement in 
performance was observed in the PT+EE+MDL100907 group compared with the PT+PBS group on both the 14th $(p=0.95)$ and 21 st $(p=0.98)$ days post-stroke. Performance of the $\mathrm{PT}+\mathrm{EE}+M D L 100907$ group was significantly worse than the PT+EE+PBS group (14th day post-PT, $p<0.0001 ; 21$ st day post-PT, $p<0.0001)$. No differences were found between the PT+PBS and PT $+M D L 100907$ groups $\left(p=0.96 ; \quad \mathrm{n}_{\mathrm{PT}+\mathrm{PBS}}=8, \quad \mathrm{n}_{\mathrm{PT}+\mathrm{EE}+\mathrm{PBS}}=5, \quad \mathrm{n}_{\mathrm{PT}+\mathrm{PBS}+}\right.$ $\left.M D L 100907=5, \mathrm{n}_{\mathrm{PT}+\mathrm{EE}+M D L 100907}=5\right)$.
A

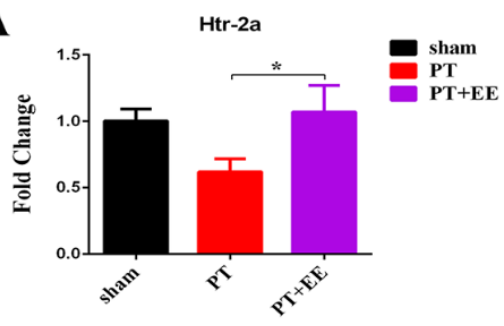

D

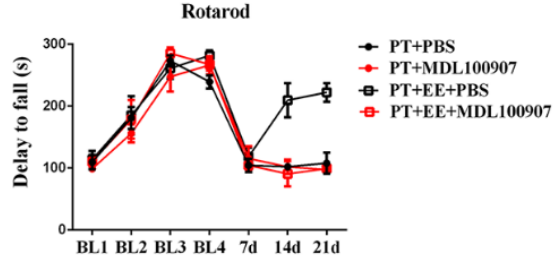

F

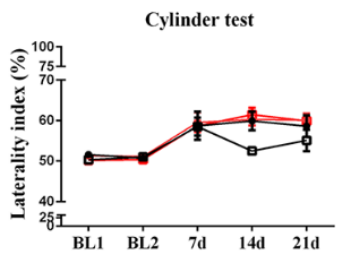

H

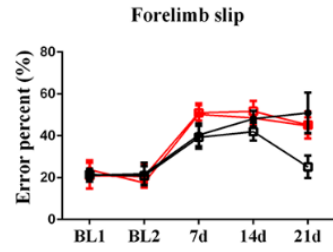

$\mathbf{J}$

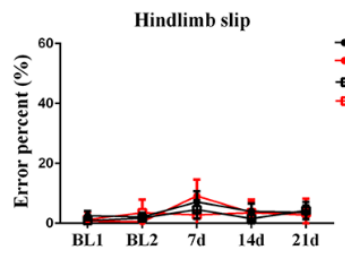

B

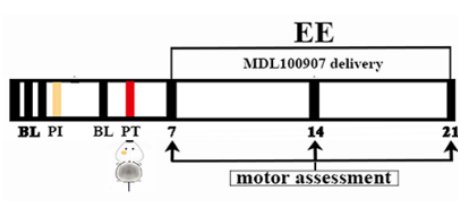

$\mathbf{E}$

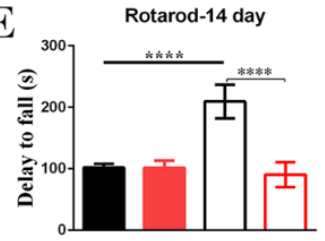

G

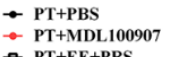
- PT+EE+PBS * PT+EE+MDL100907

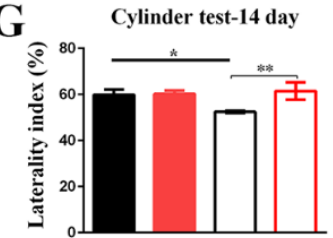

C
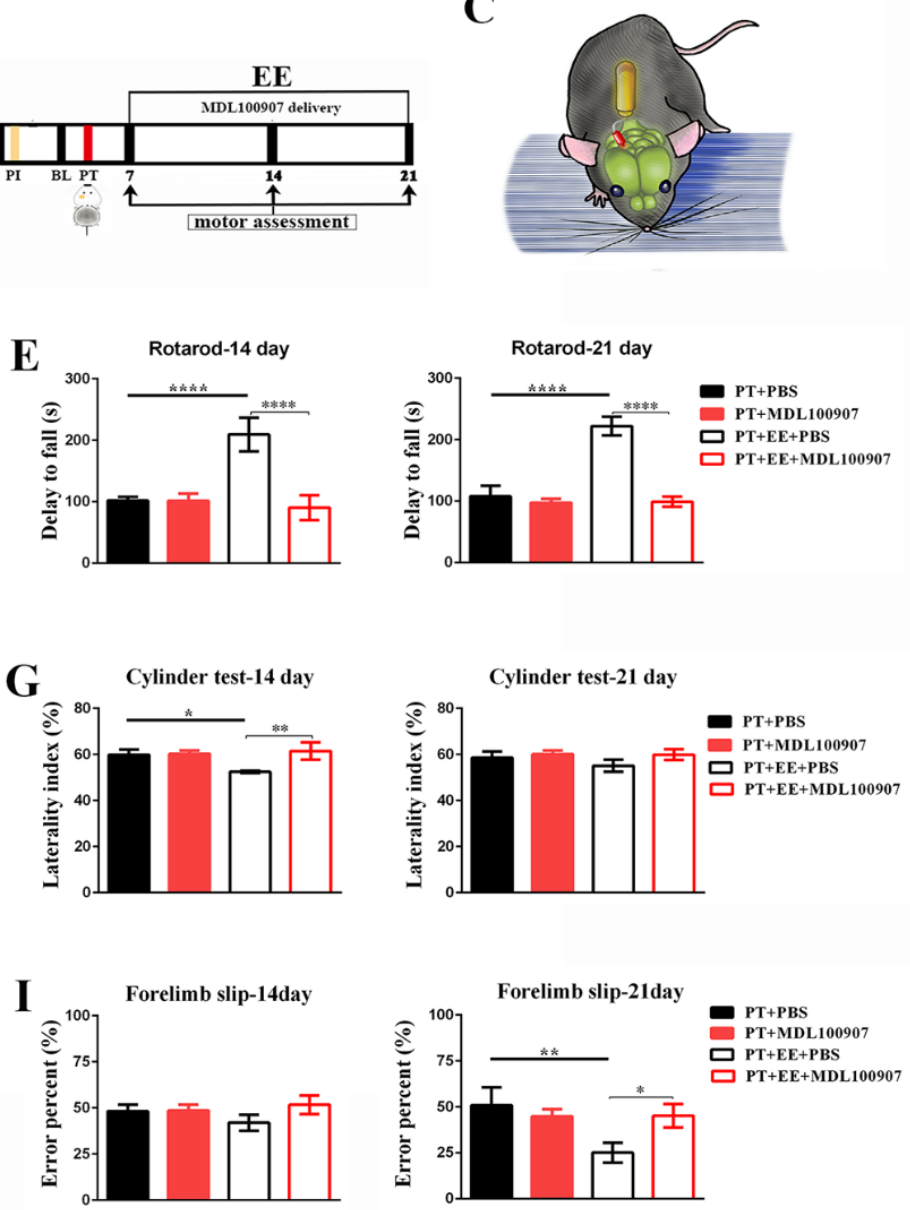

$\mathbf{K}$

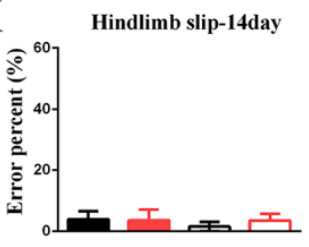

Figure 6. Effects of the 5-HT $T_{2 A}$ receptor antagonist $M D L 100907$ on motor recovery after PT. (A) Relative expression of Htr2a mRNA in each group. (B) Time course of behavioral tests after MDL100907 administration to the DCN. Motor assessments included the rotarod, cylinder and rung walking tests. BL: baseline data for rotarod. PI: osmotic pump implantation. (C) Schematic depiction of the mini osmotic pump implantation filled with MDL100907. (D) The latency to fall off the rotarod in each group. (E) Histogram of the falling off latency in each group on the 14th and 21th day after stroke. (F) The percentage of laterality in cylinder test. (G) Histogram of the laterality percentage in each group on the 14th and 21th day after stroke. (H, J) The percentage of the forelimb (H) and hindlimb (J) slips in the rung walking test. (I, K) Histogram of the error slips percentage in each group on the 14 th and $21^{\text {th }}$ day after stroke. ${ }^{*} p \leq 0.05 ;{ }^{* * *} p \leq 0.01,{ }^{* * * * *} p \leq 0.0001$. 
A

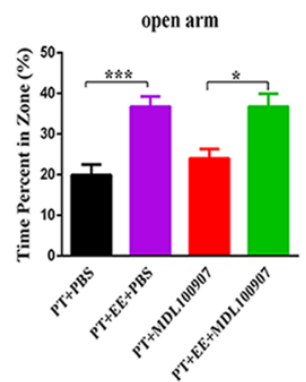

B

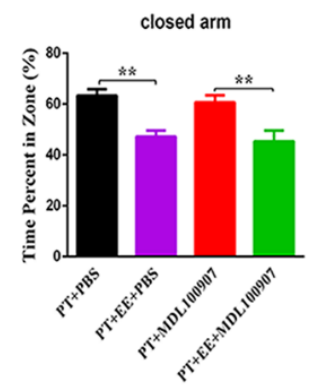

C

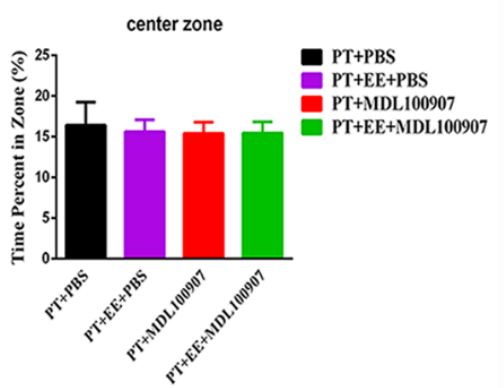

Figure 7. Effect of the 5-HT $T_{2 A}$ receptor antagonist $M D L 100907$ on anxiety-like behavior after PT. The percentage of time mice spent in the open arm (A), closed arm (B) and center zone (C) of the elevated plus maze. Data are expressed as the mean \pm SEM; ${ }^{*} p \leq 0.05$; ${ }^{* *} p \leq 0.01 ;{ }^{* * * *} p \leq 0.001$.

In the cylinder test (Fig. 6F-G). The effects of time $(F[4,100]=26.10, p<0.0001)$, treatment $(F[3,100]=3.87$, $p=0.01)$ and time $\times$ treatment interaction $\left(F_{[12,100]}=0.20\right.$, $p=0.61$ ) were analyzed using a two-way ANOVA. Results definitively demonstrated an inhibition of EE-induced recovery by $M D L 100907$. Compared to the PT+EE+PBS group, mice in the $\mathrm{PT}+\mathrm{EE}+M D L 100907$ group tended to rely more on their left forelimb $(p=0.001)$ on the 14th day post-stroke. Nevertheless, MDL100907 seemed to have no effect on PT, as the PT+MDL100907 and PT+PBS groups exhibited similar performance in limb asymmetry (14th day post-PT, $p=0.99 ; 21$ st day post-PT, $p=0.99$ ). By the 21st day post-stroke, all of the groups exhibited similar forelimb asymmetry $\left(\mathrm{n}_{\mathrm{PT}+\mathrm{PBS}}=5, \mathrm{n}_{\mathrm{PT}+\mathrm{PBS}+M D L 100907}\right.$

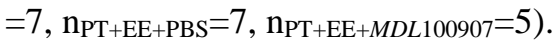

In the rung walking test (Fig. 6H-I), a two-way ANOVA with repeated measures revealed the effects of time $(F[4,100]=27.40, p<0.0001)$, treatment $(F[3,100]=3.01$, $p=0.03)$ and time $\times$ treatment interaction $(F[12,100]=1.26$, $p=0.26$ ) on forelimb placement among the three groups. No significant differences were found in the number of hindlimb slips (Fig. 6J-K). Although the number of forelimb step errors gradually decreased over time in the PT+EE+PBS group, a Tukey's multiple comparisons test revealed that the PT+EE+MDL100907 group still had a significant number of forelimb slips on the 21 st day poststroke ( $p=0.04$ ), meaning MDL100907 was able to block the effect of EE on this behavior test. Since no statistically significant difference was found between the PT+PBS and $\mathrm{PT}+\mathrm{PBS}+M D L 100907$ groups $(p=0.99)$, we may conclude that MDL100907 administration had no influence on the natural time-course of PT recovery $\left(n_{\mathrm{PT}+\mathrm{PBS}}=6, \mathrm{n}_{\mathrm{PT}+\mathrm{PBS}+M D L 100907}=7, \mathrm{n}_{\mathrm{PT}+\mathrm{EE}+\mathrm{PBS}}=6, \mathrm{n}_{\mathrm{PT}+\mathrm{EE}+}\right.$ MDL100907 $=5)$.

The 5-HT $T_{2 A}$ receptor antagonist MDL100907 has no effect on the environmental enrichment-induced attenuation of anxiety-like behavior
Because the $5-H T_{2 A}$ receptor is involved in motor recovery, we further sought to examine whether it plays a role in the EE-induced attenuation of anxiety-like behavior in the EPM test (Fig. 7). A one-way ANOVA analysis indicated that with the post-stroke administration of either PBS or MDL100907 $(\mathrm{PT}+\mathrm{EE}+\mathrm{PBS}$ and $\mathrm{PT}+\mathrm{EE}+M D L 100907$ groups, respectively), mice spent more time in the open arm (Fig. 7A), and decreased time in the closed arm of the maze (Fig. 7B), compared to the PT group (open arm: $F[3$, 29] $=11.24, \quad p<0.0001 ; \quad$ closed arm: $F[3,29]=9.07$, $p=0.0002)$. Among all groups, we found no significant differences in the amount of time spent in the center zones of the maze (Fig. $7 C ; F[3,29]=0.06, p=0.98$;. $\mathrm{n}_{\mathrm{PT}+\mathrm{PBS}}=9, \quad \mathrm{n}_{\mathrm{PT}+\mathrm{PBS}+M D L 100907}=8, \quad \mathrm{n}_{\mathrm{PT}+\mathrm{EE}+\mathrm{PBS}}=8, \quad \mathrm{n}_{\mathrm{PT}+\mathrm{EE}+}$ MDL100907 $=8$ ).

\section{DISCUSSION}

In the present study, we sought to reveal the contributions of the DCN to EE-induced post-stroke rehabilitation. We demonstrated that the ability of EE to promote post-stroke motor function was definitively dependent on the DCN activation. Furthermore, Htr2a in the DCN, the gene encoding 5-HT2A receptor, was identified as a hub gene in our PPI network. This indicates that 5-HT2A receptormediated signaling in the DCN may be necessary for the ability of EE to enhance motor function post-stroke. This work furthers our understanding of EE-induced recovery following stroke and emphasizes the appreciable role of the DCN in the process of rehabilitation.

In our work, EE resulted in the greatest enhancement in the rotarod test, which is a reliable assessment of cerebellum-dependent motor coordination (Fig. 1), compared to the cylinder or rung walking test. This disparity in the degree of improvement among different behavioral tests has led us to propose that the cerebellum may be the brain area most susceptible to EE after stroke. 
Until now, EE has been found to promote post-stroke plasticity in different brain regions $[12,13]$. The role of the cerebellum, however, has been poorly characterized [14]. Given that all outputs from the cerebellum originate from the DCN, a better understanding of the DCN's involvement in EE may help to promote more effective rehabilitation.

Interestingly, in contrast to previous studies using electrical or optogenetic stimulation of the lateral cerebellar nucleus to improve motor recovery $[6,15,16]$, DCN inhibition almost completely blocked motor recovery in EE treated mice, who performed at a level as poor as the stroke-only group. These suggest that EEinduced motor recovery after stroke was dependent on the cerebellar output pathway, and in turn, that the lack of DCN activation was likely the main cause for poor responsivity to $\mathrm{EE}$ in stroke animals. The level of cerebellar activity or metabolism has been shown to correlate with the extent of functional recovery in stroke patients $[4,5,17,18]$, our observation of poor outcomes in EE-treated mice with DCN inhibition further emphasizes the importance of post-stroke rehabilitative therapies that may modulate the DCN activation.

Furthermore, our data provides further evidence for the DCN engagement in cortical functional plasticity. As we know, the cerebellum is structurally connected to the sensorimotor cortex, and the activity and excitability of the cerebral cortex is dependent upon ascending input originating from the contralateral DCN [19]. Previous studies have demonstrated that cerebellar activation increases the plasticity of the ipsi-lesional sensorimotor cortex $[15,16,20]$. It is therefore not surprising that we observed better performance in the cylinder and rung walking tests, two sensorimotor cortex-dependent behavioral tests, in the PT+EE group as well as the $\mathrm{PT}+\mathrm{ChR} 2$ group. We should note, however, that DCN activation in EE-treated mice appeared to result in better recovery rates in the rotarod test, with less improvement in the cylinder and rung walking tests (Fig. 3). This may indicate that the DCN modulation in the cortex-dependent behavioral tests was limited. More precisely, despite normal cerebellar activity being indispensable for the EEmediated promotion of motor recovery, DCN activation may result in better motor coordination, with further improvements in fine motor ability requiring additional interventions. Future studies will seek to investigate the effects of DCN modulation on structural plasticity after $\mathrm{EE}$, including observing CPCT reconstruction by MRI and exploring the specific molecular changes underlying experience-dependent synaptic plasticity. Moreover, as far as we know, the DCN contain many neuronal subtypes, our stimulation may activate different neuronal subtypes. In the future, we will examine the different neuronal types using specific cell markers and further investigate the effect of optogenetic stimulation of selective neuronal subtypes on post-stroke recovery.

Serotonin (5-HT) has been reported to engage in a variety of physiological functions, including affective responses and cognitive processing [21]. In this study, an RNA-seq analysis revealed the hub gene, Htr2a, a gene encoding the serotonin receptor subtype 5-HT2A receptor, to be differentially expressed in the DCN in post-stroke animals receiving EE. Furthermore, we found that 5$H T 2 A$ receptor-mediated signaling was responsible for EE-induced functional recovery (Fig. 5D). Unexpectedly, an injection of the selective 5-HT2A receptor antagonist MDL100907 into the DCN only blocked EE-induced motor recovery (Fig. 6), without affecting anxiety-like behavior (Fig. 7). Indeed, considerable evidence from rodent studies has implicated the 5-HT2A receptor in behavioral changes. Unlike other 5-HT receptor subtype knockout mice [22, 23], 5-HT2A receptor-deficient mice showed more severe motor dysfunction [24-26]. Because our work has revealed an activation of the DCN after EE, as well as an ability of MDL100907 to completely block EE-induced motor recovery, we speculate that 5-HT2A receptor-mediated signaling most likely engages in the DCN activity. This hypothesis may be supported by the observation that 5-HT2A receptors are widely distributed and expressed in neuronal cell somata and dendrites of the $\mathrm{DCN}$, and 5-HT could regulate the release of many neurotransmitters such as glutamate and GABA in cerebellum [27, 28], but the exact relationship between DCN activation and 5-HT2A receptor-mediated signaling in the EE-induced post-stroke recovery needs further exploration. Thus, our future studies will employ 5-HT2A receptor-specific transgenic mice to determine the precise role of 5-HT2A receptor-expressed neurons in DCN, which may become a promising target for therapeutic modulation of cerebellar function.

In summary, we show here that the DCN activation is indispensable for EE-induced motor promotion after stroke, without being involved in the EE-induced attenuation of anxiety-like behavior. Additionally, we found that 5-HT2A receptor-mediated signaling may be responsible for DCN-dependent functional improvement in EE. These results suggest that future therapeutic approaches that aim to modulate the DCN activation may be crucial for effective rehabilitation. Moreover, further attempts to clarify the role of $5-H T 2 A$ receptor-mediated signaling in the DCN may lead to the creation of a pharmacological mimetic of the benefits of EE-induced post-stroke rehabilitation.

\section{Acknowledgments}

This study was supported by the Natural Science Foundation of China (NSFC, nos. 81472150, 81672242), 
and the key Construction Projects of Shanghai Health and Family Planning on Weak Discipline (nos. 2015ZB0401).

\section{References}

[1] Hendricks HT, van Limbeek J, Geurts AC, Zwarts MJ (2002). Motor recovery after stroke: a systematic review of the literature. Arch Phys Med Rehabil, 83:1629-37.

[2] Krishnamurthi RV, Feigin VL, Forouzanfar MH, Mensah GA, Connor M, Bennett DA, et al. (2013). Global and regional burden of first-ever ischaemic and haemorrhagic stroke during 1990-2010: findings from the Global Burden of Disease Study 2010. Lancet Global Health, 1:e259-81.

[3] Fletcher JA, Kozakewich HP, Hoffer FA, Lage JM, Weidner N, Tepper R, et al. (1991). Diagnostic relevance of clonal cytogenetic aberrations in malignant soft-tissue tumors. N Engl J Med, 324:436-43.

[4] Kim Y, Kim S, Kim J, Hong BY (2018). Modification of cerebellar afferent pathway in the subacute phase of stroke. J Stroke Cerebrovasc Dis, 27:2445-52.

[5] Takasawa M, Watanabe M, Yamamoto S, Hoshi T, Sasaki T, Hashikawa K, et al. (2002). Prognostic value of subacute crossed cerebellar diaschisis: single-photon emission CT study in patients with middle cerebral artery territory infarct. AJNR Am J Neuroradiol, 23:189-93.

[6] Elias GJB, Namasivayam AA, Lozano AM (2018). Deep brain stimulation for stroke: Current uses and future directions. Brain Stimul, 11:3-28.

[7] Yu K, Wu Y, Zhang Q, Xie H, Liu G, Guo Z, et al. (2014). Enriched environment induces angiogenesis and improves neural function outcomes in rat stroke model. J Neurol Sci, 347:275-80.

[8] Janssen H, Ada L, Bernhardt J, McElduff P, Pollack M, Nilsson M, et al. (2013). An enriched environment increases activity in stroke patients undergoing rehabilitation in a mixed rehabilitation unit: a pilot nonrandomized controlled trial. Disabil Rehabil, 36:255-62.

[9] Di Garbo A, Mainardi M, Chillemi S, Maffei L, Caleo M (2011). Environmental enrichment modulates corticocortical interactions in the mouse. Plos One, 6:e25285.

[10] Sakamoto T, Endo S (2013). Deep cerebellar nuclei play an important role in two-tone discrimination on delay eyeblink conditioning in C57BL/6 mice. Plos One, 8:e59880.

[11] Sajadi A, Provost C, Pham B, Brouillette J (2016). Neurodegeneration in an animal model of chronic amyloid-beta oligomer infusion is counteracted by antibody treatment infused with osmotic pumps. J Vis Exp, 114: 54215.

[12] Kalogeraki E, Pielecka-Fortuna J, Huppe JM, Lowel S (2016). Physical Exercise Preserves Adult Visual Plasticity in Mice and Restores it after a Stroke in the Somatosensory Cortex. Front Aging Neurosci, 8:212.

[13] Greifzu F, Pielecka-Fortuna J, Kalogeraki E, Krempler K, Favaro PD, Schluter OM, et al. (2014). Environmental enrichment extends ocular dominance plasticity into adulthood and protects from stroke-induced impairments of plasticity. Proc Natl Acad Sci U S A, 111:1150-55.
[14] Silasi G, Murphy TH (2014). Stroke and the connectome: how connectivity guides therapeutic intervention. Neuron, 83:1354-68.

[15] Cooperrider J, Furmaga H, Plow E, Park HJ, Chen Z, Kidd G, et al. (2014). Chronic deep cerebellar stimulation promotes long-term potentiation, microstructural plasticity, and reorganization of perilesional cortical representation in a rodent model. $\mathbf{J}$ Neurosci, 34:9040-50.

[16] Shah AM, Ishizaka S, Cheng MY, Wang EH, Bautista AR, Levy S, et al. (2017). Optogenetic neuronal stimulation of the lateral cerebellar nucleus promotes persistent functional recovery after stroke. Sci Rep, 7:46612.

[17] Serteser M, Ozben T, Gumuslu S, Balkan S, Balkan E (2001). Biochemical evidence of crossed cerebellar diaschisis in terms of nitric oxide indicators and lipid peroxidation products in rats during focal cerebral ischemia. Acta Neurol Scand, 103:43-48.

[18] Takuwa H, Tajima Y, Kokuryo D, Matsuura T, Kawaguchi H, Masamoto K, et al. (2013). Hemodynamic changes during neural deactivation in awake mice: a measurement by laser-doppler flowmetry in crossed cerebellar diaschisis. Brain Res, 1537:350-55.

[19] Machado A, Baker KB (2012). Upside down crossed cerebellar diaschisis: proposing chronic stimulation of the dentatothalamocortical pathway for post-stroke motor recovery. Front Integr Neurosci, 6:20.

[20] Baker KB, Schuster D, Cooperrider J, Machado AG (2010). Deep brain stimulation of the lateral cerebellar nucleus produces frequency-specific alterations in motor evoked potentials in the rat in vivo. Exp Neurol, 226:259-64.

[21] Pytliak M, Vargova V, Mechirova V, Felsoci M (2011). Serotonin receptors - from molecular biology to clinical applications. Physiol Res, 60:15-25.

[22] Köhler S, Cierpinsky K, Kronenberg G, Adli M (2015). The serotonergic system in the neurobiology of depression: relevance for novel antidepressants. J Psychopharmacol, 30:13-22.

[23] Domínguez-López S, Howell R, Gobbi G (2012). Characterization of serotonin neurotransmission in knockout mice: implications for major depression. Rev Neuroscience, 23:429-43.

[24] González-Maeso J, Weisstaub NV, Zhou M, Chan P, Ivic L, Ang R, et al. (2007). Hallucinogens recruit specific cortical 5-HT2A receptor-mediated signaling pathways to affect behavior. Neuron, 53:439-52.

[25] Halberstadt AL, Powell SB, Geyer MA (2013). Role of the 5-HT2A receptor in the locomotor hyperactivity produced by phenylalkylamine hallucinogens in mice. Neuropharmacology, 70:218-27.

[26] Halberstadt AL, van der Heijden I, Ruderman MA, Risbrough VB, Gingrich JA, Geyer MA, et al. (2009). 5HT2A and 5-HT2C receptors exert opposing effects on locomotor activity in mice. Neuropsychopharmacol, 34:1958-67.

[27] Saitow F, Murano M, Suzuki H (2009). Modulatory effects of serotonin on GABAergic synaptic 
transmission and membrane properties in the deep cerebellar nuclei. J Neurophysiol, 101:1361-74.

[28] Murano M, Saitow F, Suzuki H (2011). Modulatory effects of serotonin on glutamatergic synaptic transmission and long-term depression in the deep cerebellar nuclei. Neuroscience, 172:118-28. 